\title{
DC ELECTRICAL CONDUCTIVITY OF CHEMICALLY SYNTHESIZED CONDUCTING POLYTHIOPHENE
}

\author{
N. S. Wadatkar, S. A. Waghuley* \\ Department of Physics, Sant Gadge Baba Amravati University, Amravati \\ *Corresponding authors E-mail: nswadatkar81@gmail.com, \\ sandeepwaghuley@sgbau.ac.in
}

\begin{abstract}
Polythiophene (PTh) has attracted much attention because of its high electrical conductivity, good thermal stability, ease and high redox activity, stability and good mechanical properties. In the present work, samples of PTh were synthesized by using chemical oxidative polymerisation method with different concentration $(0.1-0.3 \mathrm{M})$ of anhydrous ferric chloride $\left(\mathrm{FeCl}_{3}\right)$ as an oxidant in aqueous medium at room temperature. The DC electrical conductivity was found in the range of $10^{-3} \mathrm{Scm}^{-1}$. In order to understand transport mechanism, temperature dependence of conductivity was investigated. The polymerization was confirmed through FTIR spectroscopy and performing XRD analysis recognized amorphous phase of the samples.
\end{abstract}

Keywords: Polythiophene; Chemical synthesis; DC electrical conductivity

\section{Introduction}

Polymers containing heterocyclic units in the backbone were found to have notable electrical conductivity and to offer increased stability and processability in both the doped and neutral states when compared with polyacetylenes. Among the many polyheterocyclics, PTh and its derivatives have received great interest due to its high conductivity, environmental stability and electrochromic properties [1]. PTh results from the polymerization of thiophenes a sulphur heterocycle that can becomes conducting when electrons are added or removed from the $\pi$ orbitals via doping. The stability of PTh in the oxidized form, its high conductivity and an interesting electrochemical behavior allow this material to use as electrode materials for electrochemical supercapacitors. The conjugated polymers have attracted a great deal of attention from many researchers, especially in the field of plastic 
electronics [2]. The conducting polymers based on heterocycles such as polythiophene (PTh), polyaniline (PANi) and polypyrrole (PPy) have attracted considerable attention in recent years due to their high conductivity, interesting electrochemical properties, and easy preparation. Several applications of these polymers are promising such as batteries, sensors, electro chromic devices, and electronic devices [3].

Three approaches to polymerization of thiophene have been reported in the literature:

(1) electropolymerization, (2) metal-catalysed coupling reactions, and (3) chemical oxidative polymerisation. Waltman et al. [4] prepared high conductivity PTh films by electropolymerization in 1983, but it is rarely used in the preparation of electro luminescent materials. Yamamoto et al.[5] reported the polycondensation of 2,5dibromothiophene catalysed by $\mathrm{Ni}$ (bipy) $\mathrm{Cl}_{2}$, and similar results were also observed by Lin and Dudek [6] in their $\mathrm{Ni}, \mathrm{Pd}, \mathrm{Co}$, and Fe catalytic system. In 1984, Yoshino et al. [7] found unsubstituted thiophene could be polymerised by ferric chloride in chloroform. The most notable property of this material such as electrical conductivity, results from the delocalisation of electrons along the polymer backbone-hence the term synthetic metals.

In the present research work, PTh samples were synthesized by chemical oxidative polymerization method by using thiophene as monomer and ferric chloride as an oxidant in deionised water. During polymerization, different concentration of $\mathrm{FeCl}_{3}$ was taken. The DC electrical conductivity measurements of different samples are made in the temperature range 303-393 K. Prepared samples were characterized through FTIR and XRD techniques.

\section{Experimental}

\subsection{Synthesis of PTh}

The AR-grade (SD-fine, India) chemicals were used in this investigation. PTh was synthesized at room temperature (303 K) by mixing thiophene 
with $\mathrm{FeCl}_{3}$ in deionised water. $\mathrm{H}_{2} \mathrm{O}_{2}$ was used to enhance the rate of reaction and also yield. After the rigorous stirring, drop-by drop monomers was added into the solution. The preliminary polymerization process was identified by the colour change (brown) of the reaction mixture. The polymerization process was allowed to constant stirring for 12-15 hrs with a magnetic

stirrer at $30{ }^{\circ} \mathrm{C}$. The resulting precipitate was collected by vacuum filtration using cellulose nitrate filter papers. The precipitate was washed with copious amounts of triply distilled water until the washings were clear and then kept for overnight at room temperature. Subsequent to this samples were sintered at $60{ }^{\circ} \mathrm{C}$ for $30 \mathrm{~min}$. In this way, different samples of PTh were prepared with different concentration (0.1-0.3 M) of $\mathrm{FeCl}_{3}$.

\subsection{Characterizations}

The samples were characterized by using FTIR and XRD techniques. The IR spectra were recorded on shimadzu (model-8201) FT-IR in the $\mathrm{KBr}$ medium at room temperature in the region $4000-500 \mathrm{~cm}^{-1}$ with $0.97 \mathrm{~cm}^{-1}$ resolution averaging 16 scans. The XRD was recorded on a Rigaku X-ray diffractometer using CuKa radiation $\left(\lambda=1.54 \mathrm{~A}^{0}\right)$. The diffractogram was in terms of $2 \theta$ in the range $10^{\circ}-70^{\circ}$.

\section{Results and Discussion}

\subsection{FTIR analysis}

The FTIR spectrum of PTh with $0.1,0.2$ and $0.3 \mathrm{M}$ of $\mathrm{FeCl}_{3}$ is displayed in Fig.1. The spectrum showed the $(\mathrm{C}-\mathrm{H})$ stretching vibration band at $2923 \mathrm{~cm}^{-1} ;(\mathrm{C}=\mathrm{C})$ stretching band at $1458-1596 \mathrm{~cm}^{-1} ;(\mathrm{C}-\mathrm{H})$ bending band at $1113 \mathrm{~cm}^{-1}$ and $(\mathrm{C}-\mathrm{S})$ bending band at $749 \mathrm{~cm}^{-1}$. The doping induced band at $1021 \mathrm{~cm}^{-1}$ was originating from the changes in the conjugated backbone due to the electron withdrawing and electron donating dopants on the polymer chain and the counter ion balancing appeared at 1113 $\mathrm{cm}^{-1}, 1309 \mathrm{~cm}^{-1}$ and $1458 \mathrm{~cm}^{-1}$ [8]. The large descending base line in the 
spectral region of $4000-2000 \mathrm{~cm}^{-1}$ was attributed due to free-electron conduction in the doped polymer.

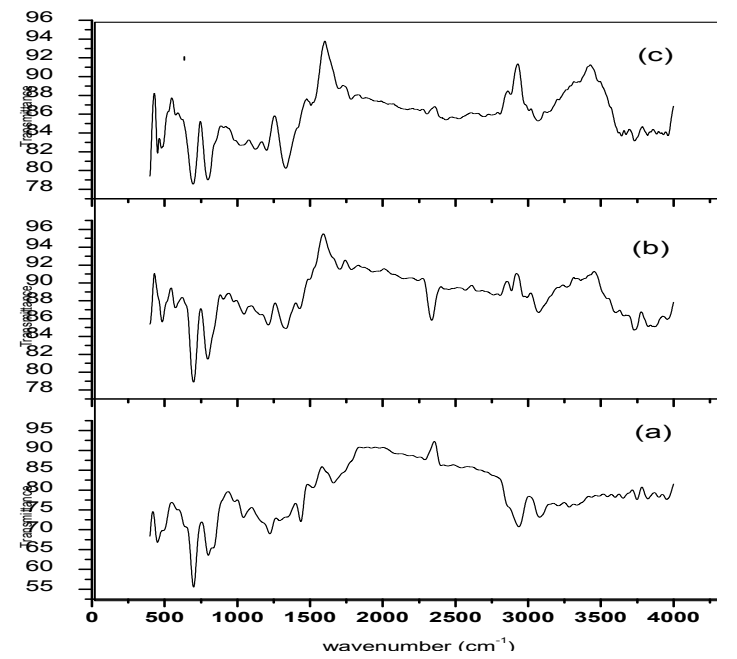

Fig. 1. FT-IR spectra of PTh samples with (a) 0.1 , (b) 0.2 and (c) 0.3M of $\mathrm{FeCl}_{3}$

This type of behaviour was not observed in undoped polymer [8]. The increasing conjugation shifted the absorption frequencies of the polymer. These results are supported to the polymerization.

\subsection{XRD analysis}

XRD analysis show that as-synthesized PTh powders are in amorphous phase as shown in Fig. 2a, b and c. The XRD spectra of sample was recorded between $2 \theta=10-70^{\circ}$. The X-ray pattern of sample shows noisy spectra. In each case, a broad peak was observed at about $2 \theta=20^{\circ}$ (Fig.2a) and $2 \theta=18^{\circ}$ (Fig. $2 \mathrm{~b}$ and $2 \mathrm{c}$ ), which is attributed to the amorphous halos nature of PTh. These peaks are due to the scattering from PTh chains at the interplanar spacing. However, the position of maximum intensity of the amorphous halos was different from each other for different PTh samples. 


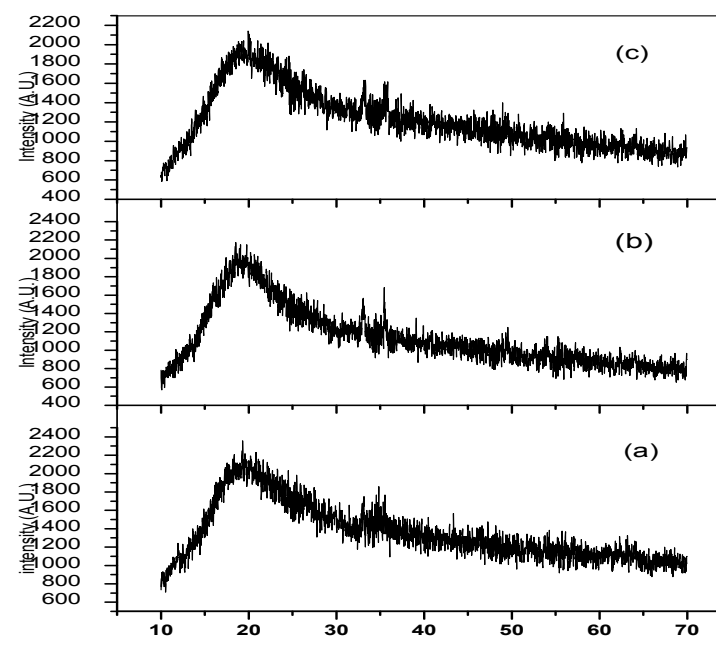

\section{Fig. 2. XRD spectra of PTh powder samples with (a) 0.1 , (b) 0.2 and}

\section{(c) $0.3 \mathrm{M}$ of $\mathrm{FeCl}_{3}$}

The average chain separation can be calculated from these maxima using the relation reported in reference [9]. The average chain separation was found to be $2.81 \mathrm{~A}^{0}$ for PTh with $0.1 \mathrm{M}$ and $3.11 \mathrm{~A}^{0}$ with 0.2 and $0.3 \mathrm{M}$ concentration of $\mathrm{FeCl}_{3}$.

\subsection{Electrical behaviour of PTh}

To investigate the charge transport mechanism in the PTh, temperaturedependent DC conductivity has been studied. Fig.3. reveals the relationship between DC electrical conductivity and temperature within the range of 303-393 $\mathrm{K}$ for PTh with $0.1,0.2$ and $0.3 \mathrm{M}$ concentration of $\mathrm{FeCl}_{3}$. From Fig. it can be seen that DC electrical conductivity increases with increase in temperature. As the concentration of oxidant $\mathrm{FeCl}_{3}$ in PTh increases, DC electrical conductivity also increases. The increased in the conductivity is attributed to an increase in charge-carrier concentration. Generally polymers are electrically insulator, but in conducting polymer backbone structure is conjugated and the existence of ionic dopant results in a delocalization of double bond. 


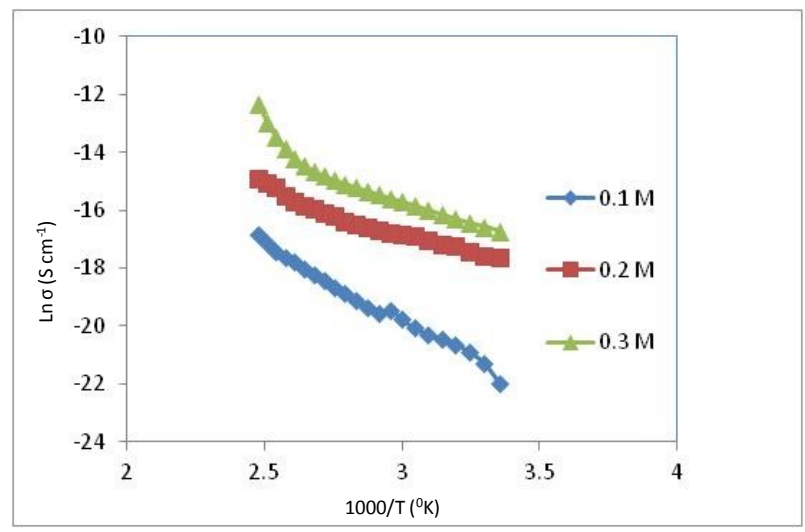

Fig. 3. Plot of $\ln (\sigma)$ as a function of $10^{3} / \mathrm{T} \mathrm{K}$ of $\mathrm{PTh}$ with $0.1,0.2$ and 0.3M concentration of $\mathrm{FeCl}$.

\section{Conclusions}

The samples were synthesized by using chemical oxidative polymerization method with anhydrous $\mathrm{FeCl}_{3}$ as an oxidant in aqueous medium at room temperature. The polymerization of thiophene was confirmed through FTIR analysis. The amorphous phase of prepared samples was recognized through XRD analysis. XRD study revealed that the average chain separation was found to be increase with oxidant concentration. The DC electrical conductivity was studied within the temperature range of 303-393 K. The entire curves are almost linear; hence the nature of the graph follows Arrhenius law. The electrical conductivity of PTh enhanced with temperature as well as concentration of oxidant.

\section{Acknowledgements}

Authors are very much thankful to Head, Department of Physics Sant Gadge Baba Amravati University, Amravati for providing necessary facilities. 


\section{References}

H. S. Nalwa, (1997). Handbook of Organic Conductive Molecules and Polymer John Wiley \& Sons, N. York.

T.A. Skotheim and J.R. Reynolds (Eds.) (2007). Handbook of Conducting Polymers, third ed., CRC Press, Boca Raton.

S.A. Waghuley, (2011) . Journal of Electron Devices, 10 ,433.

R. J. Waltman, J. Bargon and A. F. Diaz, (1983). J Phys Chem, 87, 1463.

T. Yamamoto, K. Sanechika, and A. Yamamoto, (1980). J Polym Sci Polym Lett Ed, 18, , 12.

J. W. P. Lin and L. P. Dudek, (1980). J Polym Sci Polym Chem Ed, 18, 2873.

K. Yoshino, S. Hayashi and R. Sugimoto, (1984). J Appl Phys Part 2- Lett, 23, 900 .

S.Richard, P.Gnanakan, M.Rajasekhar and A.Subramania, (2009). Int. J. Electrochem. Sci., 4, 1301.

S.A. Waghuley, S.M. Yenorkar, S.S. Yawale and S.P. Yawale, (2008) . Sens.Actuators B, $128,366$. 